\title{
The role of vertebroplasty in metastatic spinal disease
}

\author{
Julie G. Pilitsis M.D., and Setti S. Rengachary, M.D. \\ Department of Neurological Surgery, Wayne State University, Detroit, Michigan
}

\begin{abstract}
Many advances have been made in the treatment of metastatic spinal disease over the last few decades. Radiotherapy offers benefit and pain relief to many patients; however, this modality provides minimal vertebral stabilization. Surgical management consists of decompression and complex fusions. Vertebroplasty offers an adjuvant therapy to both radiotherapy and surgery by providing additional stabilization and pain relief. The results of case studies suggest that including vertebroplasty in the management of these patients is beneficial. In this article the authors review the role of vertebroplasty in metastatic spinal disease.
\end{abstract}

\section{KEY WORDS • vertebroplasty • metastasis • spine • pain • analgesia • spinal fusion}

Spinal metastases may result from nearly all malignancies, but the most frequent solid tumors spreading to the spine are breast, lung, or prostate carcinomas. ${ }^{18}$ Less frequently, renal, thyroid, or gastrointestinal carcinomas have been observed. ${ }^{18}$ The lymphoreticular malignancies, multiple myeloma and lymphoma, are also frequent causes of disseminated spinal lesions. ${ }^{18}$ Metastases account for $70 \%$ of all spinal tumors, and the lumbar spine is most frequently involved. ${ }^{25}$ Metastatic spinal lesions that cause severe back pain have a number of deleterious effects on the patient and may lead to impairment of functioning and of QOL. Furthermore, chronic pain often results in sleep loss, decreased mobility, and depression. ${ }^{13}$ Palliative treatment with bed rest, orthotics, NSAIDs, and narcotic medications has known complications. Radiotherapy provides significant pain relief but limited spinal stabilization. Vertebroplasty may be performed as a complement to radiotherapy to provide immediate pain relief and stabilization. In patients in whom surgery is contraindicated, vertebroplasty may be conducted to prevent further VB collapse and to improve pain relief. This article will describe the procedure and theoretical basis of vertebroplasty, the preoperative evaluation and indications for the procedure, and review studies in the literature in which this modality is used in the treatment of metastatic spinal disease.

\section{VERTEBROPLASTY IN METASTATIC SPINAL DISEASE}

\section{Operative Technique}

Vertebroplasty in which PMMA is used was developed in France in the late 1980s for the treatment of verte-

Abbreviations used in this paper: $\mathrm{CT}=$ computerized tomography; NSAID = nonsteroidal antiinflammatory drug; PMMA = polymethylmethacrylate; $\mathrm{QOL}=$ quality of life; $\mathrm{VB}=$ vertebral body. bral angiomas. ${ }^{7}$ Initial success with this procedure in hemangiomas has led to its use in the management of osteoporotic and osteolytic neoplastic lesions. ${ }^{1,2,7,9,23,28}$ Vertebroplasty is a minimally invasive procedure, the performance of which results in significant pain relief and spinal stabilization. The procedure is associated with limited complications.

The technique of vertebroplasty is described in various reviews ${ }^{6,8}$ Briefly, the procedure is usually performed after the injection of a local anesthetic. The patient must be able to tolerate lying in the prone position for the duration of the intervention. Fluoroscopy and/or CT guidance must be used throughout the procedure. A vertebral puncture is initiated by an approach specific to the involved vertebral level, and once positioning has been verified, a radiograph is obtained. A biopsy sample should be acquired if the primary cancer is unknown and is in a pathological fracture suspicious for metastases. The PMMA is prepared and injected once proper consistency of the compound has been reached. Injection into both the lesion and normal VB is recommended. ${ }^{8}$ The entire lesion does not need to be filled because there is no relationship between the amount of the lesion is filled and subsequent pain relief. ${ }^{6}$ On the contrary, complete filling of the lesion leads to an increased risk of cement leakage. ${ }^{8}$ The total duration of the procedure is 1 to 2 hours.

\section{Theoretical Basis of Vertebroplasty}

The authors of cadaveric studies have demonstrated that vertebroplasty improves both the strength and stiffness of abnormal bone. In one ex vivo mechanical study on the effect of cement volume on the strength in osteoporotic VBs, the investigators demonstrated that strength was restored in both the thoracic and lumbar regions when cement volumes as low as $2 \mathrm{ml}$ were injected. ${ }^{3}$ Both uni- and bilateral injections of bone cement significantly increase 
strength, ${ }^{29}$ and clinically unilateral injections have yielded pain relief. ${ }^{30}$ In another study in which the authors used a finite-element model analysis, they found that filling the VB to within only $2 \%$ of its capacity restores vertebral stiffness to within $15 \%$ of the initial value. ${ }^{20}$ It has been proposed that although restoration of strength may prevent further VB collapse, it is the restoration of stiffness that likely results in the pain resolution experienced by patients, and such relief is achieved by preventing micromotion and allowing a repair that enables healing. ${ }^{3}$ Furthermore, vertebroplasty of wedge fractures has been shown to restore flexion-extension and lateral compliance parameters to values similar to normal spine biomechanics. ${ }^{31}$

Various theories on the procedure's ability to provide pain relief have been suggested. In cases of vertebral metastases, local pain is thought to be secondary to bone fractures and the reaction of the remaining nerve structures to the tumor's mass effect. ${ }^{30}$ It is likely that a component of the vertebroplasty-related analgesia is secondary to immobilization of microfractures and reduction of mechanical forces. ${ }^{6}$ The destruction of nerve endings caused by the cytotoxic, mechanical, and vascular effects of PMMA as well as the thermal effects of polymerization, however, may also play a role in pain relief. ${ }^{6,8,30}$ Furthermore, it has been proposed that PMMA has an antitumoral effect, which may explain the rarity of local recurrence after vertebroplasty. ${ }^{8,30}$ This effect may be the result of the cytotoxicity, thermal effects, and ischemia produced by PMMA ${ }^{8,30}$ Analysis of pathological findings in patients in whom PMMA has been injected has demonstrated a macro- and microscopic rim of tumor necrosis 6 months after vertebroplasty/tumor injection, which seems to extend outside the limits of the cement. ${ }^{27}$

\section{Evaluation of Spinal Metastases}

Patients with a suspected metastatic spinal lesion must undergo a complete assessment including a physical, laboratory, and radiographical evaluation to determine the primary tumors in those in whom carcinoma was previously undiagnosed, and the extent of metastases must also be investigated. In patients with an unknown primary carcinoma, physical examination, including breast and prostate evaluations, should be performed. Routine laboratory investigations, including complete blood count, electrolytes, serum calcium and phosphate levels, liver function tests, serum prostate antigen (when indicated), and serum protein electrophoresis, should be performed. Radiological evaluation should include chest radiography, CT scanning of the head, chest, abdomen, and pelvis, and mammography in women. At our institution, oncologists and radiation oncologists are involved at an early stage to guarantee that a multidisciplinary care approach is undertaken. In patients in whom carcinoma has been diagnosed, directed testing should be performed to determine whether any additional metastases are present for prognostication.

Histological diagnosis of metastatic spinal lesions obtained from a CT-guided biopsy sampling procedure in patients who have undergone this extensive battery of testing will confirm clinical suspicion in the first biopsy attempt in $86 \%$ of cases. ${ }^{15}$ In patients who are selected for vertebroplasty, medical clearance is usually required be- cause of coexisting medical conditions. In addition, a coagulation profile should be obtained, and a neurological examination must be conducted to verify preexisting sensory and/or motor abnormalities.

\section{Indications for Vertebroplasty}

The patient who may benefit most from vertebroplasty has severe, localized, mechanical back pain related to vertebral collapse in which there is no epidural involvement. ${ }^{8}$ The pain is constant, localized, debilitating, and often worse at night. ${ }^{18}$ Attempts at achieving pain relief with nonnarcotic analgesic agents, such as NSAIDs, should be the first line of therapy. Narcotic analgesics in sustainedrelease form should be administered if NSAIDs provided no benefit. High-dose narcotics may result in somnolence, mood changes, and impairment of function. Bed rest and brace therapy are other common conservative treatment modalities. It should be noted, however, that bed rest may increase the likelihood of deep venous thrombosis in a patient in whom there is already a predisposition to this and may accelerate bone loss and muscle deconditioning. ${ }^{4}$ Brace therapy, such as the use of halo vests, although often beneficial in patients with spinal instability and/or pain, may impair QOL. Patients in whom medication and bed rest have yielded limited pain relief or whose functioning is significantly impaired by these measures should be considered for vertebroplasty.

Vertebroplasty is also useful in patients with limited anticipated survival, in poor surgical candidates, in those who have received maximum radiation doses, and in those with significant asymptomatic VB collapse secondary to a lytic lesion. ${ }^{1,8}$ The success of vertebroplasty in cases of metastatic disease has best been examined in the thoracolumbar region, although it may be used in the cervical region when surgery is contraindicated. ${ }^{8}$ Vertebroplasty should not be conducted in cases of spinal instability or in patients with spinal cord compression or epidural tumor extension who are surgical candidates. ${ }^{6,8}$ Pathological fractures resulting in pain and/or neurological symptoms, isolated metastases, radioresistant tumors, and relapse following maximum radiation doses may also be indications for surgery. ${ }^{10-12,16,19,21}$ Vertebroplasty, however, may be used as an adjuvant to surgical fusion and/or radiotherapy.

When vertebroplasty is performed in conjunction with radiotherapy, most groups recommend that vertebroplasty be undertaken prior to radiation treatment for best results. ${ }^{6,8,17}$ Murray, et al., ${ }^{22}$ have demonstrated that radiotherapeutic treatment of neoplasms is not affected by the presence of cement, nor is cement affected by radiation. In a study reported by Weill, et al., ${ }^{30}$ however, all patients in whom vertebroplasty was performed after radiotherapy failed to resolve pain experienced clear or moderate improvement.

\section{Contraindications for Vertebroplasty}

Contraindications for vertebroplasty include the following: a vertebra that has collapsed to less than one third of its original height, coagulation disorders, a patient's inability to lie prone because of medical conditions, uncertain cause of pain, and lack of immediate availability of decompressive surgery. ${ }^{6,8,30}$ Lesions causing posterior wall destruction are not an absolute contraindication to verte- 
broplasty, although the occurrence of complications appears to be increased and may pose a greater risk in the thoracic or cervical levels. ${ }^{30}$

\section{Primary Series on Vertebroplasty in Metastatic Spinal Disease}

There are four series in the literature in which investigators specifically address the use of vertebroplasty in metastatic spinal disease, as well as a limited number of other case series that include this patient group in their cohort. Deramond, et al., ${ }^{8}$ have reported their group's experience in 101 patients with metastatic spinal disease. Significant improvements in pain relief and QOL were initially demonstrated in more than $80 \%$ of their patients. ${ }^{8}$ Long-term follow-up evaluation revealed that pain control lasted in two thirds of these patients. ${ }^{6}$ Cortet, et al. ${ }^{5}$ have reported a decrease in pain within 48 hours of vertebroplasty in $97 \%$ of their 37 patients with osteolytic metastases or multiple myeloma; pain was completely absent in $13.5 \%$, significantly reduced in $55 \%$, and moderatedly reduced in $30 \%$. Beneficial effects were seen in $89 \%$ at 3 months and $75 \%$ after 6 months. Their complication rate was 2 to $3 \% .^{5}$

Kaemmerlen, et al., ${ }^{17}$ have examined vertebroplasty as an adjuvant to radiotherapy in patients with painful osteolytic metastatic lesions. Of 20 patients with 33 treated VBs, pain relief occurred in 17 in less than 48 hours, with 14 patients requiring no analgesic medication and three requiring reduced dosages of medication. No change occurred in two patients, and signs of cord compression developed in the third. No pain recurrence was demonstrated in the cohort in a mean follow-up period of 2.8 months.

Weill, et al., ${ }^{30}$ have reported a series in which they performed 40 procedures in patients with metastases. In 25 patients, vertebroplasty alone was performed. In three patients, vertebroplasty was combined with surgery, in 10 with radiotherapy, and in two with both surgery and radiotherapy. In the five patients who underwent both surgery and vertebroplasty, there was metastatic involvement of the facet joints. Both vertebroplasty and radiotherapy were performed in three patients when the cell type was determined to be radiosensitive, and the lesion surpassed the cemented portion. In seven patients, vertebroplasty was conducted after radiotherapy failed to resolve symptoms. In 26 patients, chemotherapy was administered at the time of vertebroplasty.

In their study, Weill, et al., ${ }^{30}$ defined clear pain improvement as a $50 \%$ reduction in pain medication or the replacement of narcotics with NSAIDs. Seventy-three percent had a clear improvement, $21 \%$ had a moderate improvement, and $6 \%$ had no improvement. In six of the seven patients with moderate improvement, three had extensive disease and three harbored multilacunar vertebral metastases, which are technically difficult to fill and result in leaks secondary to cortical erosion. Continued pain relief was estimated to be present in $73 \%$ of the population at 6 months and in $65 \%$ at 1 year. Patients with recurrent pain were noted to have new adjacent metastases and/or meningeal spread. In the subset of patients in whom vertebroplasty alone was performed, $74 \%$ had clear improvement, $22 \%$ had moderate improvement, and $4 \%$ had no improvement; in $76 \%$ persistent pain relief was demonstrated at 6 months. ${ }^{30}$

Barr, et al., ${ }^{1}$ included eight patients with malignancy in their cohort of patients undergoing vertebroplasty. Their primary goal of treatment was to stabilize the vertebral column. Pain relief was achieved in $50 \%$ of their patients; however, these four patients suffered advanced disease and pain that was seemingly not well localized to the treated vertebra(e). Spinal stabilization, as assessed by radiographic and clinical examination, was achieved in seven of the eight patients. ${ }^{1}$

\section{Procedure-Related Complications}

The reported vertebroplasty-related complication rate in cases of metastatic disease is approximated at $10 \% .^{8}$ This rate is higher than that observed when vertebroplasty is used to treat patients with angioma or osteoporosis $(2-5 \%$ and $1-3 \%$, respectively) and may be a result of the increased VB destruction and/or poor general condition of the cancer patient. It is important to note, however, that the long-term complication rate in the patients with metastatic disease was $1.7 \% .^{8}$

Complications of the percutaneous approach are specific to the region of the spine in which surgery is performed. At the cervical level, the carotid artery and jugular vein may be injured. At the thoracic level, a pneumothorax and/or rib fracture are possible. In both the thoracic and lumbar levels, it is essential to maintain the integrity of the internal cortex of the pedicle to lessen the incidence of cement leakage. Use of smaller-gauge needles and adequate fluoroscopic/CT guidance may limit this complication. ${ }^{8}$ It should be mentioned that if a secondary approach to the same VB is attempted, the first needle must be left in place to prevent leakage.

Leakage of PMMA occurs during injection in up to 70 to $75 \%$ of patients; however, the vast majority of these leaks are clinically unimportant. ${ }^{5,6}$ Risk factors for leakage include posterior cortical wall destruction and epidural tumors. ${ }^{6}$ If extravasation into the paravertebral soft tissues or perivertebral veins occur, injection should be immediately halted. ${ }^{8}$ Venous leakage, especially into the inferior vena cava, has been reported to result in pulmonary embolism. ${ }^{24}$ Injection of the epidural or neuroforaminal veins or extravasation into the foramina may lead to radiculopathy, which is the major risk of this procedure. ${ }^{8}$

The majority of radicular symptoms are intercostal neuralgias that require local injection. ${ }^{8}$ Radicular symptoms in the lumbar level are usually resolved by steroid therapy, but they may occasionally require decompression for resolution. ${ }^{8}$ Locally increased pain may be secondary to an inflammatory reaction to PMMA itself when no leakage has occurred. ${ }^{30}$ Patients in whom this occurs are often managed with intravenous NSAIDs and steroids for 2 to 3 days, and excellent results have been reported. ${ }^{8}$ In rare cases, leakage may induce acute spinal cord compression, especially when the posterior wall of the VB is disrupted. It is important to perform vertebroplasty in a setting in which decompression may be performed immediately if required.

\section{Prognosis After Vertebroplasty}

Although no prospective randomized controlled studies 
have been published, the authors of numerous case studies have documented the efficacy of vertebroplasty in providing pain relief and improving mobility in patients with metastatic spinal disease. These studies have documented improvement of pain in 80 to $97 \%$ of patients within 48 hours of the procedure. Barr, et al., ${ }^{1}$ reported initial improvement in only $50 \%$ of their patients; however, their indication for vertebroplasty was spinal stabilization, and their patients did not specifically have pain localized in the treated VBs. Careful patient selection must be undertaken when pain relief is the goal, as in patients with advanced disease when the source of pain may not be limited to a given vertebra. ${ }^{1}$

At 6-month follow up, 65 to $76 \%$ of patients in all studies experienced persistent pain relief. Both conventional fractionated and nonfractionated radiotherapy are associated with similar rates of pain control at 6 months. ${ }^{14,26}$ Pain relief following radiotherapy, however, is often delayed 10 to 20 days, and only minimal vertebral strengthening occurs. Unlike the delayed effects of radiotherapy, vertebroplasty provides immediate strengthening of the anterior column, which may limit painful VB collapse. ${ }^{1}$ Furthermore, vertebroplasty provides early mobility, which limits complications related to inactivity. Increased mobility and pain relief lead to improvement of QOL for these patients. Vertebroplasty is complementary to both surgery and radiotherapy and should be considered as a treatment modality in patients with metastatic spinal disease.

\section{References}

1. Barr JD, Barr MS, Lemley TJ, et al: Percutaneous vertebroplasty for pain relief and spinal stabilization. Spine 25:923-928, 2000

2. Bascoulergue Y, Duquesnel J, Leclercq R, et al: Percutaneous injection of methyl methacrylate in the vertebral body for the treatment of various diseases: percutaneous vertebroplasty. Radiology 169:372, 1988 (Abstract)

3. Belkoff SM, Mathis JM, Jasper LE, et al: The biomechanics of vertebroplasty. The effect of cement volume on mechanical behavior. Spine 26: $1537-1541,2001$

4. Convertino VA, Bloomfield SA, Greenleaf JE: An overview of the issues: physiological effects of bed rest and restricted physical activity. Med Sci Sports Exerc 29:187-190, 1997

5. Cortet B, Cotten A, Boutry N, et al: Percutaneous vertebroplasty in patients with osteolytic metastases or multiple myeloma. Rev Rhum Engl Ed 64:177-183, 1997

6. Cotten A, Dewatre F, Cortet B et al: Percutaneous vertebroplasty for osteolytic metastases and myeloma: effects of the percentage of lesion filling and the leakage of methyl methacrylate at clinical follow-up. Radiology 200:525-530, 1996

7. Deramond H, Darrason R, Galibert P: La vertébroplastie percutanée acrylique dans le traitement des hémangiomes vertébrauz agressifs. Rachis 1:143-153, 1989

8. Deramond H, Depriester C, Galibert P, et al: Vertebroplasty with polymethylmethacrylate. Technique, indications, and results. Radiol Clin North Am 36:533-546, 1998

9. Deramond H, Galibert P, Debussche C: Vertebroplasty. Neuroradiology 33 (Suppl):S177-S178, 1991

10. Dolin MG: Acute massive dural compression secondary to methyl methacrylate replacement of a tumorous lumbar vertebral body. Spine 14:108-110, 1989

11. Flatley TJ, Anderson MH, Anast GT: Spinal instability due to malignant disease. Treatment by segmental spinal stabilization. J Bone Joint Surg Am 66:47-52, 1984
12. Fraser RD, Paterson DC, Simpson DA: Orthopaedic aspects of spinal tumors in children. J Bone Joint Surg Br 59:143-151, 1977

13. Garfin SR, Yuan HA, Reiley MA: New technologies in spine: kyphoplasty and vertebroplasty for the treatment of painful osteoporotic compression fractures. Spine 26:1511-1515, 2001

14. Garmatis CJ, Chu FC: The effectiveness of radiation therapy in the treatment of bone metastases from breast cancer. Radiology 126:235-237, 1978

15. Ghelman B, Lospinuso MF, Levine DB, et al: Percutaneous computed-tomography-guided biopsy of the thoracic and lumbar spine. Spine 16:736-739, 1991

16. Gilbert RW, Kim JH, Posner JB: Epidural spinal cord compression from metastatic tumor: diagnosis and treatment. Ann Neurol 3:40-51, 1978

17. Kaemmerlen $P$, Thiesse $P$, Jonas $P$ et al: Percutaneous injection of orthopedic cement in metastatic vertebral lesions. N Engl J Med 321:121, 1989

18. Kostuik JP: Differential diagnosis and surgical treatment of metastatic spine tumors, in Frymoyer JW (ed): The Adult Spine, ed 2. Philadelphia: Lippincott-Raven, 1997, Vol 1, pp 989-1014

19. Kostuik JP, Errico TJ, Gleason TF, et al: Spinal stabilization of vertebral column tumors. Spine 13:250-256, 1988

20. Liebschner MAK, Rosenberg WS, Keaveny TM: Effects of bone cement volume and distribution on vertebral stiffness after vertebroplasty. Spine 26:1547-1554, 2001

21. Manabe S, Tateishi A, Abe M, et al: Surgical treatment of metastatic tumors of the spine. Spine 14:41-47, 1989

22. Murray JA, Bruels MC, Lindberg RD: Irradiation of polymethylmethacrylate. In vitro gamma radiation effect. J Bone Joint Surg Am 56:311-312, 1974

23. Nicola N, Lins E: Vertebral hemangioma: retrograde embolization-stabilization with methyl methacrylate. Surg Neurol 27: 481-486, 1987

24. Padovani B, Kasriel O, Brunner P, et al: Pulmonary embolism caused by acrylic cement: a rare complication of percutaneous vertebroplasty. AJNR 20:375-377, 1999

25. Posner JB: Back pain and epidural spinal cord compression. Med Clin North Am 71:185-205, 1987

26. Salazar OM, Rubin P, Hendrickson FR, et al: Single-dose halfbody irradiation for palliation of multiple bone metastases from solid tumors. Final Radiation Therapy Oncology Group report. Cancer 58:29-36, 1986

27. San Millán Ruíz D, Burkhardt K, Jean B, et al: Pathology findings with acrylic implants. Bone 25 (Suppl 2):85S-90S, 1999

28. Tampieri D, Weill A, Melanson D, et al: Percutaneous aspiration biopsy in spine lytic lesions. Indications and techniques. Neuroradiology 33:43-47, 1991

29. Tohmeh AG, Mathis JM, Fenton DC, et al: Biomechanical efficacy of unipedicular versus bipedicular vertebroplasty for the management of osteoporotic compression fractures. Spine 24: 1772-1776, 1999

30. Weill A, Chiras J, Simon JM, et al: Spinal metastases: indications for and results of percutaneous injection of acrylic surgical cement. Radiology 199:241-247, 1996

31. Wilson DR, Myers ER, Mathis JM, et al: Effect of augmentation on the mechanics of vertebral wedge fractures. Spine 25: $158-165,2000$

Manuscript received October 11, 2001.

Accepted in final form November 2, 2001.

Address reprint requests to: Julie G. Pilitsis, M.D., Department of Neurosurgery, Wayne State University, University Health Center Suite 6E, 4201 St. Antoine, Detroit, Michigan 48201. email: jpilitsis@neurosurgery.wayne.edu. 